\title{
Thermography for polymers film blowing
}

\author{
by P. G. Berardi ${ }^{1}$, G. Cuccurullo ${ }^{1}$ and L. Di Maio ${ }^{2}$ \\ ${ }^{1}$ Department of Mechanical Engineering, University of Salerno, 84084 Fisciano, Italy \\ ${ }^{2}$ Department of Chemical and Food Engineering, University of Salerno, 84084 Fisciano, Italy
}

\begin{abstract}
Polymer temperature distribution in film blowing was investigated by means of an IR thermography system sensing in the window between 2 and $5 \mu \mathrm{m}$. Since in this band polymers behave as selective emitters, the emission and reflection of the radiation are bulk and surface phenomena. Thus, a preliminary study on the film radiative behaviour is presented in order to characterise the thermography readout on semitransparent sheets. Then, experiments on film blowing extrusions have been performed with polypropylene on a pilot plant in order to measure the on-line film temperature distribution for different working conditions.
\end{abstract}

\section{Introduction}

Polymeric film production by film blowing technology is a common operation in polymer processing: it essentially consists of film formation from a tubular which is blown and cooled in air and drawn by nip rolls, see Fig. 1. Films for packaging and plastic bags represent typical products. The cooling rate of the polymeric bubble is controlled by means of an air ring positioned at the die exit. In this operation the thermal aspect plays an important role because the cooling rate affects the final film quality [1][2][3][4][5]. In facts the bubble geometry (diameter and thickness), the polymeric chain orientation (i.e. the physical properties) and the film temperature distribution strongly depend on working conditions (polymer mass rate, drawing, blowing and cooling). In particular they determine the location of the region in which the solidification takes place along the path between die exit and nip rolls (freeze line).

Due to its ability of performing non-invasive measurements, it is tempting to use thermography for detecting on-line molten polymers film temperature. Unfortunately, unlike of what happens for opaque materials where the radiation is a superficial phenomenon, in the semitransparent materials a portion of the radiation comes from the bulk. Since molten polymers behave like semitransparent materials, as they present a peak of absorption at $3.43 \mu \mathrm{m}$, characteristic of the $\mathrm{C}-\mathrm{H}$ bond [6], a preliminary study for the characterisation of the radiative exchange involving semitransparent media under film blowing conditions has been carried out, then experiments are performed on a pilot plant with PP films.

\section{The radiative model}

When a radiometer looks to the film surface in the blowing process, in particular in the region behind the frost line, the bubble can be treated as if it were two flat parallel plates. For characterising the film radiative exchange, it is sufficient to consider a single slab [7], as sketched in Fig.2, with a radiometer looking at right angle to the surface.

The radiation field inside the slab can be obtained by solving the local radiant energy balance equation for a dielectric medium:

$$
\frac{d I_{\perp, v}(x)}{d x}=k_{v}\left(n_{v}^{2} I_{b b, v}-I_{\perp, v}\right)
$$

where: $I_{\perp, v}$ is the normal spectral intensity; $I_{\mathrm{bb}, v}=I_{\mathrm{bb}, v}(T(x))$, is Plank's function, $T(x)$ being the local temperature; $k_{v}$ and $n_{v}$ are the spectral absorption coefficient and the refractive index of the media. 
The radiative behaviour of the slab material is described by two intensive parameters: the spectral absorption coefficient and the refractive index, both weakly dependent on temperature; in the following they will be assumed temperature independent.

The formal solution to the radiative equation can be conveniently obtained with respect to the forward and backward intensity components, $\underset{I^{\perp, 4}}{+}$ and ${ }_{\mathrm{I}^{\perp, 4}}^{-}$:

$$
\begin{gathered}
I_{\perp, v}^{+}(\xi)=\Gamma_{I^{\perp, v}}^{+}(\xi=0) \exp (-\xi)+n_{v}^{s} \int_{0}^{\xi} I_{d d, v}\left[T\left(\xi^{+}\right)\right] \exp \left(-\left(\xi-\xi^{+}\right)\right) d \xi^{+} \\
\left.\left.I_{\perp, v}^{-}(\xi)=I_{\perp, v}^{-}(\xi=L) \exp (\xi,-\xi)\right]+n_{v}^{s} \int_{3}^{\xi}\right\lrcorner I_{, v}[T(\xi)] \exp (-(\xi-\xi)) d \xi
\end{gathered}
$$

where $\xi=w_{x}$ is the optical path; $\xi_{\perp}={ }_{k v}$ is the optical thickness, ${ }_{\Gamma}$ being the slab thickness. The first term on the right-hand represents the contribution due to the wall intensity, attenuated for the absorption, while the second is the emitted intensity, which, in turn, is attenuated: the attenuation of the boundary intensity is gradually replaced with the radiation emitted and attenuated along the beam.

The radiative boundary conditions are:



where: $\rho_{\perp, y}$ and $\tau_{\perp, y}=1-\rho_{\perp, y}$ are the normal spectral reflectivity and transmittivity of the interface between the slab and the environment; ${ }_{\mathrm{I}^{j, y}}^{j x t}$ is the external intensity impinging on the slab.

For an optically smooth surface, the spectral reflectivity is related to the complex refractive indices of both the slab and the surrounding medium by means of the Fresnel relations. For a dielectric slab embedded in air, one obtains:

$$
b_{1,4}=\frac{\left({ }_{15}-1\right)^{5}}{\left({ }_{w}+1\right)^{s}}
$$

The problem being linear, the radiative field can be split in two terms: the former related to the slab emission, the latter to the slab absorption, i.e. with reference to the case of "hot" and "cold" slab [8], respectively.

\section{hot slab}

In this case the radiative balance equation remains the same, while the $\mathrm{BCs}$ are simplified because no external radiation is incident.

The radiative field can be obtained once ${ }_{\mathcal{L}^{+, i}}^{+}(\xi=0)$ and ${ }_{\mathcal{L}^{\perp, 4}}^{-}\left(\xi=\xi_{J}\right)$ are known:

$$
\begin{aligned}
& I_{\perp, V}^{+}(\xi=0)=\left[\rho_{\perp, V} /\left(1-\rho_{\perp, V}^{s} T_{\perp, V}{ }^{s}\right)\right]\left(J_{\perp, V}^{+}+\rho_{\perp, V} T_{\perp, V} J_{\perp, V}^{-}\right) \\
& I_{\perp, V}^{-}\left(\xi=\xi_{\perp}\right)=\left[\rho_{\perp, V} /\left(1-\rho_{\perp, V}^{s} T_{\perp, V}{ }^{s}\right)\right]\left(\rho_{\perp, V} T_{\perp, V} J_{\perp, V}^{+}+J_{\perp, V}^{-}\right)
\end{aligned}
$$

where:

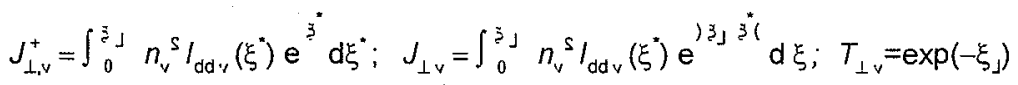

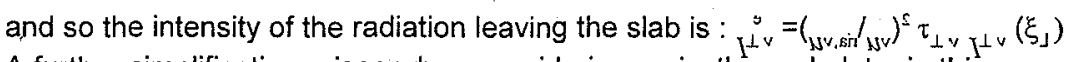

A further simplification arises when considering an isothermal plate; in this case the slab spectral emittance can be defined and is given by:

$$
\boldsymbol{e}_{\perp, \mathrm{v}}=I_{\perp v}^{\rho} /\left(n_{\mathrm{v}, \mathrm{Bir}} I_{\mathrm{dd} \mathrm{v}}\right)=\left(1-\rho_{\perp v}\right)\left[1-T_{\perp v}\right] /\left[1-\rho_{\perp v} T_{\perp, v}\right]
$$

The slab emittance turns out to depend on slab material and thickness. 


\section{cold slab}

In this case the radiative boundaries equations remain the same, while the radiative balance is simplified because the slab doesn't emit, i.e. $(\xi)=0$. The radiative field can be now easily obtained and the spectral transmittance of the stab turns out to be:

$$
t_{\perp v}=I_{\perp v}^{s} / I_{\perp v}^{\text {gx }}=\left(1-\rho_{\perp v}\right) T_{\perp v} /\left[1-\rho_{\perp v} T_{\perp v}\right]
$$

The transmittance is the only bulk parameter to specify, since the spectral absorptance is equal to the spectral emittance for isothermal slab and the reflectance can be derived by an overall energy balance.

\section{double slab system}

Having in mind the bubble film processing, two identical isothermal slabs are considered. Recalling that the film temperatures are higher than the surrounding ones, the radiative contribution of this latter can be neglected, thus the external intensity coincides with the emitted backward slab intensity. The radiation leaving the system toward the radiometer is due to: the forward radiation emitted from the front slab; the backward radiation emitted from the front slab and the forward radiation emitted by the rear slab, which undergo multiple reflections and transmissions between the two slabs. The emittance of the system is:

$$
e_{\perp v \text {.twoa ade }} / e_{\perp v}=1+t_{\perp v}\left(1+\rho_{\perp v}\right) /\left(1-\rho_{\perp v}\right)=1+t_{\perp v} /\left(1-\rho_{\perp v}\right)
$$

As expected the radiation leaving the system composed of two slabs is greater than one referred to a single slab.

\section{hot PP slab}

As an application of the above analysis, the radiation emitted by a single isothermal PP slab is considered. For this material the spectral absorption coefficient is well known [9], see Fig. 3: its spectra exhibits a peak at about $3.5 \mu$ with a strong variation in the interval 2.5 $4.5 \mu \mathrm{m}$, so that the material cannot be considered a gray medium within this interval. Concerning the reflectivity, i.e. the refraction index, for polymers it is typically constant throughout the whole spectrum with a characteristic value of $\rho_{\perp v}=0.07$.

Fig. 4 shows the spectral radiation emitted by a single PP slab at $100^{\circ} \mathrm{C}$ with different thickness. The limit behaviours of optically thick and thin slab are quite evident for high and low values of thickness, respectively. In the optically thick approximation, the leaving radiation is related only to surface phenomena; in particular the emittance turns out to be: $e_{\perp v}=1-\rho_{\perp v}=0.93$. In the optically thin approximation, the beam is attenuated very little and the radiation field is dominated by the emission. The slab absorbs and emits little so the radiation emitted by the slab tends to vanish. Far from these two limiting cases, the slab exhibits a semitransparent behaviour depending on the wavelength: the curves resemble the fingertip of the absorption coefficient.

\section{IR radiometer measurement}

With the above clues for guidance, the radiometer response in measuring temperature of semitransparent sheets will be investigated. In the following an ideal infrared radiometer is considered, that is an instrument whose sensor receives the same energy leaving the object and delivers a signal independent of the wavelength. For a real IR radiometer the knowledge of the spectral transfer function is needed, but the basic analysis is not affected. The two cases of isothermal and non-isothermal slab will be analysed. 


\section{isothermal slab}

In Fig. 5, attention is focused on the response of an ideal IR radiometer with a typical SW sensitivity band $(2<\lambda<5 \mu \mathrm{m})$, looking both to a single and to two PP sheets for different thickness and temperature. With reference to a single slab, it can be seen that the radiation detected by the radiometer increases with increasing thickness, showing a strong variation in the range between $0.05 \mathrm{~mm}$ and $2 \mathrm{~mm}$ and a weak dependence on the slab temperature. For a system composed of two slabs at the same temperature, the emissivity is greater than the one corresponding to a single slab; in particular they tend to coincide in the limit of optical thin, $e_{\perp \mathrm{RAD}} \rightarrow 0$, and thick approximations, $e_{\perp \mathrm{RAD}} \rightarrow 1-\rho_{\perp}=0.93$; in the latter case the radiometer is no more able to look at the backward slab. The emissivity chart presented in Fig. 5 allows to obtain the slab temperature once the slab thickness is known; the emissivity dependence on temperature, although slight, requires a suitable calibration.

\section{non isothermal slab}

A further problem, unfortunately, arises with IR radiometer looking at non-isothermal semi-transparent slabs with a temperature gradient across the thickness. For the sake of simplicity a single hot slab is considered.

For a given temperature profile, the intensity leaving the slab can be calculated, but not closed form; indeed, the slab emittance looses its meaning because there is not a unique temperature to evaluate the $b b$ intensity. However, the intensity leaving the slab is converted by the IR radiometer into a single temperature for each radiometer emissivity setting: a curve relating the emissivity and the thermography temperature readout is thus obtained.

In addition, if the thickness is known, the emissivity chart for isothermal slabs can be used, to give a further curve relating the emissivity and temperature. The intersection between the two curves will give a single value for temperature and emissivity. This temperature, $T_{R A D}$, will represent a reference temperature for the slab: it is the temperature at which an isothermal slab emits the same radiation of the non-isothermal one [10]. The reference temperature, of course, is related to the slab temperature distribution: it will have to coincide with the surface temperature for slab thickness increasing, i.e. approaching to the opaque body limit and to be an opportune average of the slab temperature for thickness decreasing, i.e. approaching the transparent slab limit.

In Fig. 6, the temperature readout is reported for PP slabs with different thickness. Parabolic temperature distribution is assumed inside the slab with surface temperature at $100^{\circ} \mathrm{C}$ and maximum one at $115^{\circ} \mathrm{C}$. As expected, for optically thick slabs, $T_{R A D}$ recovers the surface temperature, while, for decreasing thickness, the temperature increases till to reach a value of $110^{\circ} \mathrm{C}$, value very close to the average temperature across the slab.

\section{Experimental results}

\section{experimental setup}

The production of tubular film was achieved by making use of a film blowing extrusion laboratory system. It is constituted of an extruder (BRABENDER L/D $=400 / 20$ ) equipped with an annular die (diameter $20 \mathrm{~mm} \times$ thickness $0.5 \mathrm{~mm}$ ) and a HAAKE take-up system. The extruder temperature profile, from the hopper to the head, was $230-220-210^{\circ} \mathrm{C}$ and the other operative parameters are listed below and correspond to typical working conditions for real blown film production plants: extruder screw speed, 20 R.P.M. (i.e. $25 \mathrm{~g} / \mathrm{min} \mathrm{ca}$ ); bubble take-up velocity, from 2.5 to $10 \mathrm{~m} / \mathrm{min}$; bubble draw up ratio, from 5 to 20 ; bubble blow-up ratio, fixed at 1.5. The above set of conditions is such that the location of the frost line does not change along the machine direction: the distance between extrusion head and the frost line was approximately $55 \pm 5 \mathrm{~mm}$. The thickness of final film (from off line measurements), depending on draw up ratio, was approximately ranging from 0.01 to $0.045 \mathrm{~mm}$. 
The film thermal response, in the region between die to chill roll, was detected by means of an AGEMA 870 IR system.

\section{experimental tests}

Several experimental tests have been performed at the same polymer mass rate, i.e. for extruder velocity of $20 \mathrm{rpm}$, with different draw ratio: 5, 10, 15 and 20 , corresponding to takeup velocities of $2.5,5,7.5$ and $10 \mathrm{~m} / \mathrm{min}$; the off line thickness are: $45,25,15$ and $10 \mu \mathrm{m}$, respectively.

The picture in Fig. 7 gives the apparent blackbody temperatures with reference to the above tests, as an example. It can be noted that the isotherms are not horizontal, i.e. the film does not exhibit the expected axial symmetry. This is due both to mass rate oscillations and, mostly, to imperfections of the gap of extrusion die which in turn determine transversal thickness variations.

In Fig. 8 the apparent blackbody temperature profiles in the machine direction are reported for two different runs; it is evident that the repeatability is not good as observed before. However, the curves at different DR exhibit the same behaviour:

- in the first region, confined between the die exit and freeze line, the temperature decreases rapidly; here the polymer is still molten and undergoes a strong thickness decreasing

- in the second region, i.e. the frost line zone, the temperature is quite constant, due to the crystallization process

- in the last region, the temperature shows a slight monotonic decay; here the polymer attained the solid phase and the thickness is practically uniform.

- the rapid change in slope in the transition between the first and the second region, i.e. the crystallization starting, occurs at the same axial location

With reference to the last point, the frost line height depends essentially on the cooling air flow; in particular, increasing the air velocity reduces the frost line height [11]. The experiments having been carried out without changing the cooling conditions and the blow up ratio, the position of the frost line is approximately constant.

\section{data reduction}

From literature, the temperature of starting crystallization is relatively well known and depends essentially on the draw ratio; for our working parameters, typical values are between 75 and $95^{\circ} \mathrm{C}$, the upper values being related to the lower draw ratio; a rough measure by means of thermocouples in the crystallization region confirmed the above range. The following crystallization temperatures, obtained as average values on different runs, are assumed: $T_{\text {cryst }}=90.3,86.2,82.7$ and 80.4 for DR $5,10,15$ and 20 , respectively.

The above assumption allowed reducing the apparent $b b$ temperature into temperature profiles, see Fig. 9. This procedure is strictly meaningful in the crystallization region and can be extended reasonably in the downstream provided that the thickness is practically the same. Indeed, in the molten region, the thermography temperature readout represents a sort of average value, because the inner core layer will be hotter than the surface and the thickness undergoes a strong variation. The features highlighted with reference to the three regions still hold.

Finally, the values of the radiometer emissivity just obtained are placed on the emissivity chart of Fig. 5, which is referred to the ideal radiometer. Recalling that, in the temperature range under examination, the curves for the ideal radiometer are practically independent of the temperature, they collapse into a single one; for real radiometers the same behaviour can be foreseen. The experimental points, as expected, doesn't fall on the curve related to the ideal radiometer, but they exhibit the same functional dependence with respect to the thickness. 


\section{Conclusions}

Quantitative IR thermography can be applied to temperature detection in film blowing operation, but care must be taken due to the semitransparent behaviour of polymers. In particular sensible results can be obtained in the case of isothermal media provided that both the thickness and spectral radiative properties of media are known; while for a nonisothermal medium the the IR temperature readout will lead to an estimation of the temperature level through the film. In addition, to perform a quantitative analysis, detailed information on the spectral transfer function of the IR system as a function of the wavelength are needed.

First experiments revealed the existence of three different regions, the second one being interested by crystallization process, and that the crystallization starts at the same axial location, easily detectable by rapid change in slope. The assumption of crystallization temperatures allowed to obtain temperature profiles along the machine direction.

Further development of this work will be essentially concerned with enhancing the experimental work: adopting spectral filters on the radiometer (or different radiometers, in principle), in order to obtain further information about the inner film temperature distribution; testing different working conditions, such as higher DR, and different polymers for correlating "dynamic" crystallization effect to the temperature distribution; controlling the bubble stability with reference to the repeatability.

\section{REFERENCES}

[1] AGASSANT (J. F.), AVENAS (P.), SERGENT (J.) and CARREAU (P.J.) - Polymer processing. Hanser publ., 1991.

[2] RAUWENDAAL (C.) - Polymer extrusion. Hanser publ., 1986.

[3] AMMIRATI (C. C.), DI MAIO (L.), CUCCURULLO (G.) and ACIERNO (D.) - Heat exchange in film casting: modelling and experimental determination. PPS -12, Sorrento, 1996, pp. 313-314.

[4] BARQ (P.), HAUDIN (J. M.) and AGASSANT (J. F.) - Isothermal and anisothermal models for cast film extrusion. Int. Polymer Processing, n. 14, 1992, pp.334-349.

[5] D'HALEWYU (S.), AGASSANT (J.F.) and de MAY (Y.) - Numerical simulation of the cast film process. Polymer Engineering and science, Vol.30, n. 6, 1990, pp. 335-342.

[6] DEWITT (D. P.) and NUTTER (G. D.) - Theory \& practice of radiation thermometry. Wiley \& sons, 1989.

[7] OBENDRAUF (W.), LANGEKER (G. R), FRIESENBICHLER (W.) - Temperature Measuring in Plastics Processing with Infrared Radiation Thermometers. Int. Polymer Processing XIII, Hanser Pub., Munich, 1998.

[8] VISKANTA (R.) and ANDERSON (E. E.) - Heat Tranfer in Semitransparent Solids. Advances in Heat Tranfer, Academy press, Vol. 11, 1975, pp. 317-387.

[9] HAJJI (N.) and SPRUIELL (J. E.) - Radiation Pyrometry on Semitransparent Sheets I: Gray Media. Polymer Engineering and Science, Vol. 34., 1994.

[10] HAJJI (N.) and SPRUIELL (J. E.) - Radiation Pyrometry on Semitransparent Sheets II: Media with Wavelength Dependent Absorption Coefficient. Polymer Engineering and Science, Vol. 34., 1994.

[11] WHITE (J. L.) and CAKMAK (M.) - Advances in Polymer Technology, vol. 8, n. 1, 1988, pp. $27-61$. 
http://dx.doi.org/10.21611/qirt.1998.042

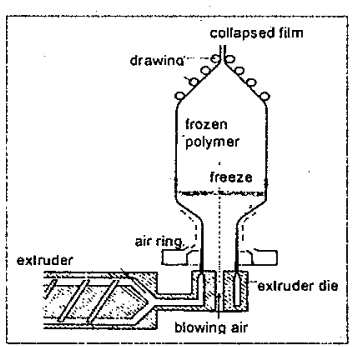

Fig.1. Film blowing

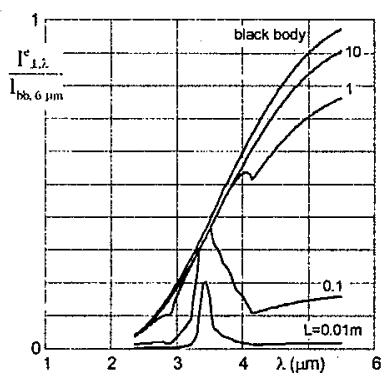

Fig. 4. The emitted radiation of one PP slab

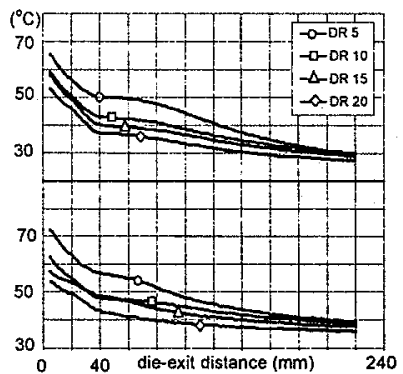

Fig. 7. Apparent black body temperature profiles

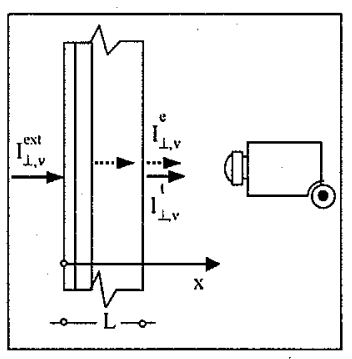

Fig. 2. The radiative model

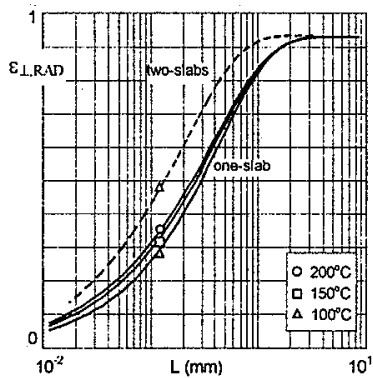

Fig. 5. The emissivity chart for PP slabs

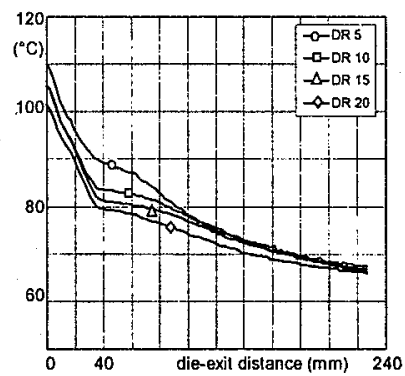

Fig. 8. Temperature readout profiles

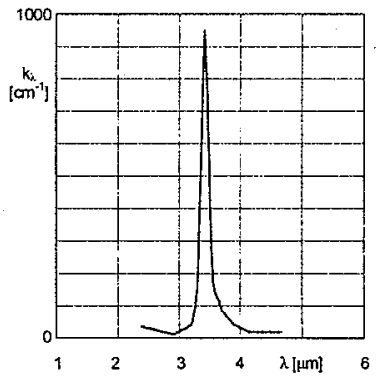

Fig. 3. Absorption spectrum

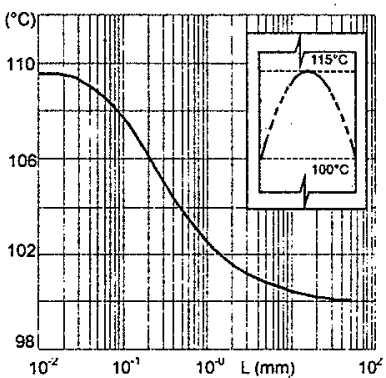

Fig. 6. Temperature for one non isothermal slab

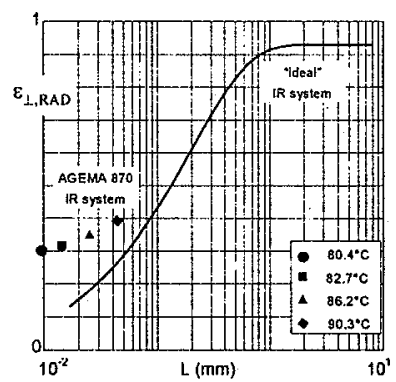

Fig. 9. The emissivity chart for PP film blowing


Fig. 10. Thermography maps at different $D R$ 